\title{
Human Atheromatous Plaque Extracts Induce Tissue Factor Activity (TFa) in Monocytes and also Express Constitutive TFa
}

\author{
Thomas W. Muhlfelder, Vic toria Teodorescu, Jacob Rand, Alan Rosman, \\ J ulian Niemetz
}

From the Bronx VA Medic al Center and The Mount Sinai School of Medic ine, New York, USA

\section{Summary}

Tissue factor activity $(\mathrm{TFa})$ is a major activator of the coagulation cascade and may play a role in atheroma-induced thrombosis. Monocyte-macrophages (MO-MF) generate considerable quantities of $\mathrm{TFa}$ when stimulated by a variety of inducers. To test the hypothesis that MO could be induced by atheromatous plaque to generate TFa, plaque extracts obtained from patients with obstructive atheromatous disease were used. These extracts were also assayed for constitutive TFa. The constitutive activity was variable from extract to extract but could be very high, up to $250 \mathrm{U} \mathrm{TFa}$. The TFa induced in MO could be also very high, up to $200 \mathrm{U}$ (i.e. $1 / 5$ of the TFa of full strength rabbit brain thromboplastin). These findings point to a major role for MO-MF TFa in the induction or thrombosis by atheromatous plaque.

\section{Introduction}

Arterial thrombosis is a well known complication of atheromatous plaques and is triggered by plaque fissure resulting in strokes, myocardial infarction and other thrombotic events (1-5). Reocclusion of a vessel which had been recently permeabilized either by lysis or mechanically, is a vexing and dangerous event which may occur even in the presence of vigorous anticoagulation $(6,7)$. The origin of this prothrombotic activity is incompletely elucidated. Tissue factor (TF) is a lipidated integral membrane-bound glycoprotein whose activity (TFa) is a powerful promoter of blood coagulation.

TFa complexes with factor VII/VIIa to hydrolytically activate factor $\mathrm{X}$ and factor IX leading to the formation of thrombin and subsequently a fibrin clot $(8,9)$. TF mRNA and TF antigen have been detected in the adventitia of arteries (10). However, the adventitia is isolated from blood circulation. TFa has been induced in vitro in endothelial cells (11), and TF mRNA has been induced in the muscularis after balloon injury of the vessel (12).

Two circulating blood elements, MO and platelets, could be implicated in plaque-induced thrombogenesis. MO-MF may be induced to generate TFa, and have a well-established thrombogenic activity (13). Mo TFa may be induced by a variety of agents, such as bacterial endotoxin (lipopolysaccharide) $(13,14)$, antigen-antibody complexes (15), complement fragments (16), and lipoproteins $(17,18)$. It is noteworthy that MO TFa generation occurs not only in vitro but also in vivo as has been demonstrated not only in peritoneal MF (13) but also in peripheral blood MO in rabbit and human $(19,20)$. Moreover, it has been recently reported that MO of patients with coronary ischemic syndromes bear TF antigen (21).

Correspondence to: Dr. Thomas W. Muhlfelder, Bronx VA Medical Center, 9A Hematology, Kingsbridge Rd., Bronx NY 10468, USA - Tel.: +17185849000; FAX Number: +17185791635
Furthermore, Tipping et al. have shown that MF teased out of atheromatous plaques have $\mathrm{TFa}$ (22). More recently, TFa has been demonstrated within atheromatous plaques obtained from coronary arteries at the time of surgery (33). Thiruvikraman et. al. have reported TF in human atherosclerotic plaques by digoxigenin-labeled coagulation factors VIIa and X. TF was within the extracellular lipid-rich core, macrophages, smooth muscle cells, adventitia and endothelium (34). Ardissino et al. have shown TF antigen and activity in coronary vessels were lowest in patients with stable angina and became progressively higher in patients with unstable angina and myocardial infarction respectively (38).

Platelets also play a role in atheroma-induced thrombosis. Platelets adhere to atheromatous vessel lesions (3). Furthermore, a monoclonal antibody inhibiting platelet function facilitated repermeabilization of injured vessels in dogs and reduced mortality in patients undergoing angioplasty (23-25).

In this report we provide evidence that atheromatous plaque extracts may contain substantial amounts of $\mathrm{TFa}$, and that the atheromatous plaques may induce TFa generation in MO. Therefore specific inhibitors of $\mathrm{TFa}$ and $\mathrm{TFa}$ generation by MO may be an appropriate form of anticoagulation during angioplasty.

\section{Materials and Methods}

Plaque material was obtained sterily during surgery from atheromas of aorta, carotid or leg arteries and was handled in endotoxin-free containers. Prior to extraction plaques were assessed qualitatively to be either soft or hard (and calcified). Plaques were extracted with $3 \mathrm{ml}$ of $0.9 \% \mathrm{NaCl}$ in a hand held glass tissue homogenizer with 8-10 strokes under a sterile hood. Non-dispersed material was sedimented for $5 \mathrm{~min}$ at $1 \times \mathrm{g}$ and discarded. The extracts were either tested at once or frozen at $-70^{\circ} \mathrm{C}$, and tested at a later date. Freezing did not affect the results. The extracts were tested for constitutive TFa, or studied as inducers of MN-TFa.

Peripheral blood MN were prepared as previously described either from the patient from whom the atheromatous specimen was obtained or a blood group compatible donor; this did not alter the results. Briefly whole blood was anticoagulated with $4 \%$ sodium citrate. The blood was diluted by $50 \%$ with phosphate buffered saline (PBS) without Ca or Mg (Grand Island Biologicals, NY), layered on Ficoll-Hypaque (Pharmacia, Piscataway, NJ) and centrifuged at $400 \times \mathrm{g}$ for $40 \mathrm{~min}$ at 14 degrees $\mathrm{C}$. The MN layer was collected and washed twice with PBS, without $\mathrm{Ca}, \mathrm{Mg}$; the last wash was with PBS-1\% human albumin (26). Cells were then suspended in RPMI 1640 (Grand Island Biologicals N.Y.). MN preparations contained 25\% MO on average. For some experiments fetal calf serum (FCS), heated at $60^{\circ} \mathrm{C}$ for $1 \mathrm{~h}$, was added to RPMI to achieve a final concentration of $12 \%$ in the incubation mixture.

Reagents and components partaking in incubation mixtures where MOgenerated TFa was assessed, were handled sterily and were endotoxin-free. $\mathrm{MN}\left(3 \times 10^{6} /\right.$ tube $)$ were incubated with either atheromatous plaque extract and RPMI 1640, or endotoxin $0.05 \mu \mathrm{g} / \mathrm{ml}$ (Difco, Detroit, MI) as a standard inducer of TFa, or RPMI 1640 alone as negative control. After $16 \mathrm{~h}$ incubation at 


\section{Monocyte TFa Generation Induced by Plaque Extract}

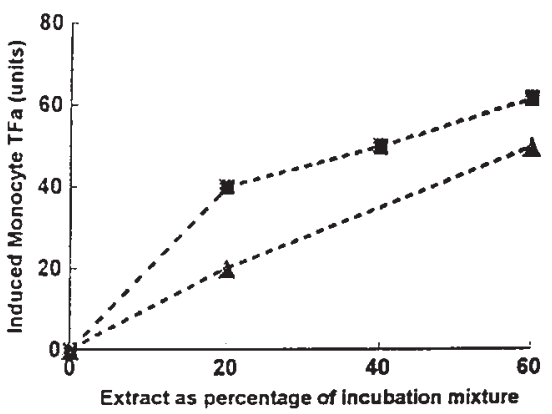

Fig. 1 Dose-dependent increase of plaque-induced MN TFa. The symbols represent two separate experiments

$37^{\circ} \mathrm{C}$, MN were washed by suspension in $3 \mathrm{ml}$ of $0.9 \% \mathrm{NaCl}$, and sedimented at $400 \times \mathrm{g}$ for $10 \mathrm{~min}$ at $14^{\circ} \mathrm{C}$ and the supernate discarded. The washing step was repeated and the cells resuspended in initial volume of $0.9 \% \mathrm{NaCl}$, were sonically disrupted before being tested for TFa. Testing for TFa was done by the one stage test and the results compared to those of 5 dilution of rabbit brain thromboplastin, which at full strength had an arbitrarily assigned activity of $1000 \mathrm{U} / \mathrm{ml}$ (14). MN incubated in RPMI had a TFa, which was always below $0.5 \mathrm{U}$.

Neutralization of constitutive and induced TFa was carried out with monoclonal anti-TFa (mab TFa) kindly provided by Dr. Yale Nemerson, Mount Sinai School of Medicine (33). Specimens containing TFa were incubated for $30 \mathrm{~min}$ at $37^{\circ} \mathrm{C}$ at antibody concentrations of $8 \mu \mathrm{g} / \mathrm{ml}, 42 \mu \mathrm{g} / \mathrm{ml}$ or with control media (PBS-1\%albumin) and the residual TFa was then measured.

Platelet aggregation was performed with the platelet Aggregation Profiler, model PAP-4 (Bio/Data Corporation, Horsham, PA). Platelet-rich plasma

Table 1 Constitutive TFa was determined on plaque extract. Plaque-induced MO TFa was determined on MNs $\left(3 \times 10^{6}\right)$ incubated for $16 \mathrm{~h}$ with plaque extract. Thereafter, MNs were washed, disrupted and tested for TFa

\begin{tabular}{lcl}
\hline & $\mathrm{N}$ & Induced TFa U \\
\hline Soft plaque & 31 & $15(0-75)$ \\
Hard plaque & 7 & $13(0-52)$ \\
RPMI & - & $<0.5$ \\
\hline & $\mathrm{N}$ & Constitutive TFa U \\
\hline Soft plaque & 30 & $22(0-250)$ \\
Hard Plaque & 5 & $3(0-7)$ \\
\hline
\end{tabular}

Table 2 Extracts of six consecutive plaques were compared for TFa inducer activity. MNs were incubated with plaque extract-RPMI, or with plaque extract-RPMI enriched with heat-inactivated FCS. Constitutive TFa was also assayed and compared. Note: absence of enhancement of endotoxin-induced TFa by FCS

\begin{tabular}{llll}
\hline Sample & Induced (Plaque) & $\begin{array}{l}\mathrm{TFa}(\mathrm{U}) \\
\text { Induced (Plaque + FCS) }\end{array}$ & Constitutive \\
\hline 1 & 18 & 25 & 27 \\
2 & 33 & 84 & 24 \\
3 & 75 & 200 & 14 \\
4 & 3 & 3 & 33 \\
5 & 9 & 130 & 6 \\
6 & 1 & 19 & 9 \\
Endotoxin & 9 & 5 & - \\
RPMI & $<1$ & $<1$ & - \\
\hline
\end{tabular}

(PRP), 300,000 platelets/ $\mu$ l, and platelet-poor plasma (PPP) were prepared from blood-group compatible donors and used to test the ability of atheromatous extract to induce aggregation. Epinephrine, ADP and collagen (Bio/Data Corp.) were used at final concentrations of $10 \mu \mathrm{M}, 2 \mu \mathrm{M}$ and $19 \mathrm{mg} / \mathrm{ml}$ respectively, and induced appropriate platelet aggregation in PRP. Fifty $\mu$ l of saline plaque extract were added to $450 \mu \mathrm{l}$ of PRP in the cuvette and platelet aggregation was measured.

Total protein, triglycerides, total cholesterol and HDL-C were measured by standard laboratory methods on plaque extracts. The total protein was measured by the biuret method; triglycerides and total cholesterol were quantitated in the Boehringer Mannheim/Hitachi 747 analyzer. HDL-C was quantitated on the Beckman Synchron CX.

Numerical data were expressed as the mean \pm standard error of the mean. As the TFa did not follow a normal distribution, non-parametric tests were used. The Mann-Whitney-U test was used for TFa from different samples. Paired data (i.e. TFa activity from the same samples) were evaluated with the Wilcoxon test. The relationships among induced and constitutive TFa and other factors were analyzed with the Spearman rank correlation (Zar, 1984). Results were considered statistically significant if the p values were less than 0.05 . Statistical calculations were performed using SPSS for Windows (SPSS Inc., Chicago, Illinois).

\section{Results}

\section{Plaque Induced TFa in MO}

To determine whether plaque extracts may induce TFa generation in MO, autologous, or blood-group-compatible MN were incubated with plaque extracts and after $16 \mathrm{~h}$ incubation the TFa generated was assayed. Some extracts induced considerable activity, while others did not (Table 1). The induced activity was dependent on the concentration of extract added (Fig. 1).

Enrichment of MN culture media with FCS enhanced generation of MO TFa by plaque extract. The generated TFa of 6 consecutive extracts were compared. Addition of $12 \%$ FCS in the MN-plaque extract incubation mixture amplified TFa generation in some, but not all of the samples. Enhancement was up to 19 -fold and as much as $200 \mathrm{U}$ TFa per $\mathrm{ml}$ was generated in the presence of FCS. The enhancement was significant at $p=0.04$ level. These results show that under some conditions the induced TFa could be very high, in most instances severalfold higher than the TFa induced by endotoxin. However, absence of enhancement by FCS in 2 atheroma-extract samples, absence of enhancement by FCS in the endotoxin specimen, and absence of enhancement by FCS in RPMI indicate that FCS by itself was devoid of inducer TFa activity (Table 2).

\section{Constitutive TFa}

The constitutive TFa of 35 atheromatous plaque extracts was examined. Thirty extracts were obtained from soft plaques. The TFa was quite variable ranging from 0 to $250 \mathrm{U}$. The mean was $22 \mathrm{U}$. The activity of the remaining 5 extracts obtained from hard, calcified plaques was lower ranging from 0 to $7 \mathrm{U}$ of $\mathrm{TFa}$ (Table 1).

To establish that the procoagulant activity was $\mathrm{TFa}$, plaque extracts were incubated with mabTF and the remaining activity after $30 \mathrm{~min}$ was assayed. The neutralization of TFa was dose-dependent (Fig. 2). These results indicate that the major procoagulant activity is indeed due to TFa.

To discriminate between induced and carried-over constitutive $\mathrm{TFa}$ in incubations of $\mathrm{MN}$ with plaque extract, $\mathrm{MN}$ were washed prior to assay for TFa. Constitutive TFa was not carried over to the incubation mixtures of plaque extract with MO. Indeed, when specimens with high 
Table 3 Comparison of constitutive and induced TFa. All samples with high induced $\mathrm{TFa}(>30 \mathrm{U})$ were compared with the constitutive TFa in the corresponding extract. Similarly, all samples with high constitutive TFa $(>30 \mathrm{U})$ were compared with the corresponding induced $\mathrm{TFa}$

\begin{tabular}{lccc}
\hline $\begin{array}{l}\text { High induced with corresponding } \\
\text { constitutive TFa }\end{array}$ & $\begin{array}{l}\text { High constitutive with corresponding } \\
\text { induced TFa }\end{array}$ \\
\hline Induced & Constitutive & Constitutive & Induced \\
\hline 52 & 1 & 45 & 3 \\
62 & 31 & 250 & 3 \\
52 & 2 & 47 & 24 \\
200 & 14 & 50 & 18 \\
33 & 1 & 37 & 14 \\
84 & 24 & 33 & 3 \\
130 & 6 & & \\
\hline
\end{tabular}

(over $30 \mathrm{U}$ ) induced $\mathrm{TFa}$ were compared with their corresponding constitutive $\mathrm{TFa}$, the induced activities were up to 52 times greater. Similarly, plaque extracts with the highest constitutive activity also correlated poorly with the induced activity (Table 3 ). These findings indicate that the induced $\mathrm{TFa}$ could not be carried-over TFa.

$\mathrm{TFa}$ of plaque extracts were compared with their lipid and protein content. No correlation was found between cholesterol, HDL, and triglyceride concentrations on one hand and TFa, constitutive or induced, on the other. However, a negative correlation was observed between total protein concentration and constitutive TFa $(\mathrm{p}=0.03)$ (Table 4).

\section{Platelet Aggregation}

Ability to aggregate platelets in PRP was demonstrated by 5 of 9 extracts. Maximum aggregatory activity ranged from 7 to $48 \%$. In comparison, standard agonist-induced aggregations were: epinephrine, 76\%; ADP, 70\%; and collagen 70\%. There was no correlation between plaque-induced platelet aggregation and $\mathrm{TFa}$, whether induced or constitutive (Table 5).

\section{Disc ussion}

We have demonstrated constitutive $\mathrm{TFa}$ in many atheromatous plaque extracts which were obtained surgically from patients with advanced atheromatous disease. These results were recorded in abstract form $(27,28)$. The TFa we report could be quite high (up to $250 \mathrm{U}$ ), particularly in soft plaques but it was variable. The variable level of constitutive TFa from extract to extract may be due to testing of plaques at different stages of development. Indeed calcified plaques had lesser quantities of constitutive TFa. Additionally, the putative presence of inhibitors in some plaques may also be responsible for the observed lack of TFa in some specimens.

Importantly, we report that many plaque extracts induced generation of $\mathrm{TFa}$ in peripheral blood $\mathrm{MO}$ which could be more than 50 times

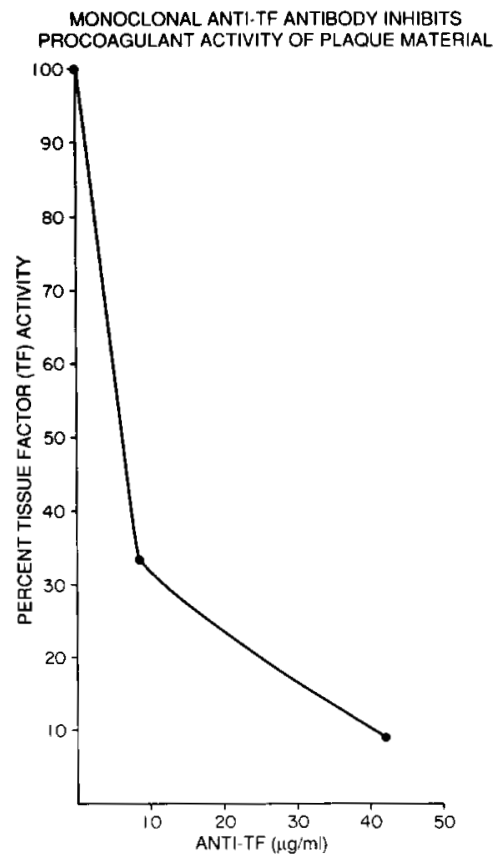

Fig. 2 Dose-dependent neutralization of plaque extract TFa by mab TFa

Table 5 Platelet aggregation by plaque extract was measured on platelet-rich plasma. Aggregation was compared to constitutive and induced TFa of these extracts

\begin{tabular}{lcc}
\hline $\begin{array}{l}\text { \% aggregation } \\
\text { of platelets }\end{array}$ & Constitutive TFa & Induced TFa \\
\hline 48 & 10 & 11 \\
34 & 2 & $<1$ \\
21 & 2 & 6 \\
12 & 47 & 24 \\
7 & 7 & 1 \\
0 & 2 & $<1$ \\
0 & 2 & 1 \\
0 & 7 & 6 \\
0 & 50 & 18 \\
\hline
\end{tabular}

higher than the respective constitutive TFa (Table 3). We propose that resident MF as well as MO attracted to the plaque may be induced to generate TFa. As MF may represent up to $60 \%$ of the cells in a plaque (29), considerable amounts of TFa could be generated

Attempts to identify inducer of MO-TFa led us to quantify plaque proteins and lipids and correlate their concentration with TFa. Neither constitutive nor induced TFa correlated well with the concentration of cholesterol, HDL-C or triglycerides in the plaque extract. However there was a negative correlation between constitutive $\mathrm{TFa}$ and total protein concentration. At least three possible explanations exist for this

\begin{tabular}{llllrrl}
\hline TFa & & & \multicolumn{2}{l}{ Plaque constituents $(\mathrm{mg} / \mathrm{dl})$} & \\
Sample & Constitutive & Induced & Protein & TG & TC & HDL-C \\
\hline$\# 1$ & 27 & 18 & 200 & 4 & 16 & 6 \\
$\# 2$ & 24 & 33 & 300 & 22 & 37 & 7 \\
$\# 3$ & 14 & 75 & 200 & 8 & 6 & 2 \\
$\# 4$ & 33 & 3 & 100 & 12 & 26 & 6 \\
$\# 5$ & 6 & 9 & 600 & 35 & 45 & 9 \\
$\# 6$ & 9 & 1 & 300 & 5 & 6 & 3 \\
\hline
\end{tabular}

Table 4 Constitutive and induced $\mathrm{TFa}$ are compared with protein, triglyceride (TG), total cholesterol (TC) and HDL-cholesterol (HDL-C) 
negative correlation. 1. Poor co-extraction of proteins and constitutive TFa. 2. Co-extraction of an inhibitor of TFa. 3. TFa and protein concentration may peak at different times in the evolution of an atheromatous plaque.

There is strong evidence that the high concentration of MO-MF within the plaque is a result of chemotactic factors secreted from cellular elements of the vascular wall. Indeed MO chemotactic factors including Monocyte Chemotactic Protein (MCP) and other cytokines have been demonstrated in atheromatous plaques (30). Poon et al. showed that monocyte chemotactic activity was generated and secreted by cultured rat aortic vascular smooth muscle cells in response to platelet-derived growth factor-BB. Antisense oligonucleotides and antibody to JE/MCP-1 were able to completely block monocyte migration (35). Zeiher et al. were able to demonstrate the up-regulation of MCP-1 by cultured human umbilical vein endothelial cells by inhibition of basal nitric oxide production (36).

Platelets have been shown to adhere to atheromatous lesions in vitro (37). In our experiments atheromatous plaque was able to induce platelet aggregation in 5 out of 9 cases. This may be of considerable importance in thrombogenesis since it has been reported previously that platelets powerfully enhance endotoxin-induced MO TFa, through the mediation of 12-HETE (26). Moreover, activated platelets may directly induce MO TFa generation, possibly by platelet p-selectin (32). The clinical relevance of these observations may be related to the improved outcome of some patients undergoing coronary angioplasty who were treated with mab to IIb/IIIa platelet glycoprotein receptors (25).

We propose that plaque TFa is at least in part due to MF-MO TFa. The TFa is available on the surface of the intact MF-M0, but it also may be released (31) or may be a remnant of disrupted MF.

The following scenario is proposed: Plaque is ruptured, circulating $\mathrm{MO}$ are recruited by the plaque and are induced by yet unknown factors to generate TFa. Platelets attracted to the plaque enhance the generation of TFa by MO-MF. This cascade of events, which need not peak simultaneously, may be responsible for fibrin deposition over the fissure of the atheromatous plaque.

\section{Acknowledgments}

We wish to thank Yale Nemerson M.D. for providing mabTF and polyclonal TF antibody; Ezra Levy Ph. D for measuring the plaque lipids; Ralph Williams for preparing the tissue sections for immunologic study.

\section{References}

1. Constantinides P. Plaque fissures in human coronary thrombosis. J Atheroscler Res 1966; 6: 1-17.

2. Davies MJ, Thomas AC. Plaque fissuring: The cause of acute myocardial infarction, sudden ischemic death, and crescendo angina. Br Heart J 1985; 53: 363-73.

3. Fuster V, Badimon L, Badimon JJ, Chesebro JM. The pathogenesis of coronary artery disease and the acute coronary syndromes: Parts 1 and $2 . \mathrm{N}$ Engl J Med 1992; 326: 242-50, 310-8.

4. Levin DC, Fallon JT. Significance of the angiographic morphology of localized coronary stenoses: histopathologic correlations. Circulation 1982; 66: 316-20.

5. Ross R. Atherosclerosis: A defense mechanism gone awry. Amer J Path 1993; 143: 987-1002.

6. Johnson DE, Hinohara T, Selmon MR, Braden LJ, Simpson JB. Primary peripheral arterial stenoses and restenoses excised by transluminal atherectomy: a histopathologic study. J Am Coll Cardiol 1990; 15L: 419-25.
7. Schwartz 1, Bourassa MG, Lesperance J, Aldridge HE, Kasim F, Salvatori VA, Henderson M, Bonan R, David PR. Aspirin and dipyridamole in the prevention of restenosis after percutaneous transluminal coronary angioplasty. N Engl J Med 1988; 318: 1714-9.

8. Nemerson Y. Tissue factor and hemostasis. Blood 1988; 71: 1-8.

9. Osterud B, Rapaport SI. Activation of Factor IX by the reaction product of tissue factor and Factor VII: additional pathway for initiating blood coagulation. Proc Natl Acad Sci USA 1977; 74: 5260-4.

10. Wilcox JN, KM Smith, SM Schwartz, Gordon D. Localization of tissue factor in the normal vessel wall and in the atherosclerotic plaque. Proc Natl Head Sci USA 1989; 86: 2839-43.

11. Colucci M, Balconi G, Lorenzet R, Pietra A, Locati D, Donati MB, Semeraro $\mathrm{N}$. Cultured human endothelial cells generate tissue factor in response to endotoxin. J Clin Invest 1983; 71: 1893.

12. Marmur JD, Rossikhina M, Guha A, Fyfe B, Friedrich V, Mendlowitz M, Nemerson Y, Taubman MB. Tissue factor is rapidly induced in arterial smooth muscle after balloon injury. J Clin Invest 1993; 91: 2253-9.

13. Niemetz J, Fani K. Role of leukocytes in blood coagulation and the generalized Shwartzman reaction. Nature New Biol 1971; 232: 247-8.

14. Niemetz J. The coagulant activity of leukocytes: Tissue factor activity. J Clin Invest 1972; 51: 307-13.

15. Rothberger H, Zimmerman TS, Spielgelberg HL, Vaughn JM. Leukocyte procoagulant activity: Enhancement of production in vitro by $\operatorname{IgG}$ and antigen-antibody complexes. J Clin Invest 1977; 59: 549-57.

16. Muhlfelder T, Niemetz J, Kreutzer Beebe D, Ward PA, Rosenfeld S. C5 chemotactic fragment induces leukocyte production of tissue factor activity-link between complement and coagulation. J Clin Invest 1979; 63: $147-50$.

17. Levy GA, Schwartz BS, Curtiss LK, Edgington TS. Plasma lipoprotein induction and suppression of the generation of cellular procoagulant activity in vitro: Requirements for cellular collaboration. J Clin Invest 1981; 67: 1614-22.

18. Schwartz BS, Levy GA, Curtiss LK, Fair DS, Edgington TS. Plasma lipoprotein induction and suppression of the generation of cellular procoagulant activity in vitro: Two procoagulant activities are produced by peripheral blood mononuclear cells. J Clin Invest 1981; 67: 1650-8.

19. Thiagarajan P, Niemetz J. Procoagulant-tissue factor activity of circulating peripheral blood leukocytes. Results of in vivo studies. Thromb Res 1980; 17: $891-6$.

20. Osterud B, Glaegstad T. Increased tissue thromboplastin activity in monocytes of patients with meningococcal infection. Thromb Haemost 1983; 49 (1): 5-7.

21. Leatham PM, Bath W, Tooze JA, Camm AJ. Increased monocyte factor expression in coronary disease. Br Heart J 1995; 73: 10-3.

22. Tipping PA, Malliaros J, Holdsworth SR. Procoagulant activity expression by macrophages from atheromatous vascular plaques. Atheroscl 1989; 79: 237-43.

23. Coller BS, Peerschke EL, Scudder LE, Sullivan CA. A murine monoclonal antibody that completely blocks the binding of fibrinogen to platelets produces a thrombasthenic-like state in normal platelets and binds to glycoproteins IIb and/or IIIa. J Clin Invest 1983; 72: 325-38.

24. Gold HK, Coller BS, Yasuda T, Saito T, Fallon JT, Guerrero JL, Leinbach RC, Ziskind AA, Collen D. Rapid and sustained coronary artery recanalization with combined bolus injection of recombinant tissue-type plasminogen activator and monoclonal antiplatelet GPIIb/IIIa antibody in a canine preparation. Circulation 1988; 77: 670-7.

25. The EPIC Investigators. Use of a monoclonal antibody directed against the platelet glycoprotein IIb/IIIa receptor in high-risk coronary angioplasty. N Engl J Med 1994; 330: 956-61.

26. Lorenzet R, Niemetz J, Marcus NJ, Broekman MJ. Enhancement of mononuclear procoagulant activity by platelet 12 -hydroxyeicosatetraenoic acid. J Clin Invest 1986; 78: 418-23.

27. Chmielewska J, Sundin G, Lundell K, Lothgren A, Ericksson B, Risberg B, Andersson LO. Thrombogenicity of human atherosclerotic plaques. Thromb Haemost 1993; 69 (6): 1013 (Abstr.). 
28. Muhlfelder T, Teodorescu V, Esseesse I, Niemetz J. Procoagulant activity of atheromatous plaques from human arteries. Thromb Haemost 1993; 69 (6): 1176 (Abstr.).

29. Jonasson L, Holm J, Skalli O, Boudjers CT, Hansson AV. Regional accumulation of T cell, macrophages and smooth muscle cells in human atherosclerotic plaque. Arteriosclerosis 1986; 6: 131-8.

30. Valente AJ, Rozek MM, Sprague EA, Schwartz CJ. Mechanisms in intimal monocyte-macrophage recruitment. A special role for monocyte chemotactic protein-1. Circulation (suppl) 1992; 86: III-20-5.

31. Muhlfelder TW, Khan I, Niemetz J. Factors influencing the release of procoagulant-tissue factor activity from leukocytes. J Lab Clin Med 1978; 92 : 65-72.

32. Celi A, Pellegrini G, Lorenzet R, De-Blasi A, Ready N, Furie BC, Furie B. P-selectin induces the expression of tissue factor on monocytes. Proc Natl Acad Sci USA 1994; 91 (19): 8767-71.

33. Marmur JD, Thiruvikraman SV, Fyfe BS, Guha A, Sharma SK, Ambrose JA, Fallon JT, Nemerson Y, Taubman MB. Identification of active tissue factor in human coronary atheroma. Circulation 1996; 94 (6): 1226-32.
34. Thiruvikraman SV, Guha A, Roboz J, Taubman MB, Nemerson Y, Fallon JT. In situ localization of tissue factor in human atherosclerotic plaques by binding of digoxigenin-labeled factors VIIa and X. Laboratory Investigation 1996; 75 (4): 451-61.

35. Poon M, Hsu WC, Bogadanov VY, Taubman MB. Secretion of monocyte chemotactic activity by cultured rat aortic smooth muscle cells in response to PDGF is due predominantly to the induction of JE/MCP-1. Am J Path 1996; 149 (1): 307-17.

36. Zeiher AM, Fisslthaler B, Schray-Utz B, Busse R. Nitric oxide modulates the expression of monocyte chemoattractant protein 1 in cultured human endothelial cells. Circulation Research 1995; 76 (6): 980-6.

37. van-Zanten GH, de-Graaf S, Slootweg PJ, Heijnen HF, Connolly TM, de-Groot PG, Sixma JJ. Increased platelet deposition on atherosclerotic coronary arteries. J Clin Invest 1994; 93 (2): 615-32.

38. Ardissino D, Merlini PA, Ariens R, Coppola R, Bramucci E, Mannucci PM. Tissue-factor antigen and activity in human coronary atherosclerotic plaques. The Lancet 1997; 349: 769-71.

Received June 16, 1997 Accepted after resubmission September 14, 1998

DISCLAIMER Published materials reflect the authors' opinions only, not those of the editor of the journal or the publisher. Neither the editor nor the publisher assume any warranty for the correctness of the opinions or representations made by the authors.

No conclusion as to whether it is a question of registered or non-registered trade marks may be drawn from the mentioning of trade names or marks in this journal. 